\title{
Application of Op-amp Fixators in Analog Circuits
}

\author{
R. Rohith Krishnan ${ }^{\# 1}$, S. Krishnakumar ${ }^{* 2}$ \\ ${ }^{\#}$ Department of Electronics, S.T.A.S, M.G. University Regional Centre \\ Edappally, Kochi, Kerala, India \\ ${ }^{1}$ rohithpunnoor@gmail.com \\ 2 drkrishsan@gmail.com
}

\begin{abstract}
Nullor elements have applications not only in analog behaviour modeling but also in analog circuit design and analysis. Fixator-norator pair, the emerging tool in analog design is a combination of a nullor and sources. A method for the realization of fixator-norator pair is discussed in this paper. Application of fixator-norator pair into a circuit makes it possible to perform the AC and DC designs in a linear like way. Fixator fixes a critical biasing spec at the design, whereas the pairing norator finds the value of power conducting components or DC sources that meets the design. A scaling amplifier design, an active load design and a CMOS differential amplifier design are provided as examples to demonstrate the procedure and the methodology.
\end{abstract}

Keyword- Fixator-norator pair, Nullor, Op-amp, Analog circuits.

\section{INTRODUCTION}

Source allocation and biasing design are the major steps in the design of analog circuits. The process becomes harder as the length of the circuit increases. Nonlinearity is one of the problems that make the design process difficult and time consuming. A linear network is much easier to design as it obeys superposition principle. Thus, a method that performs linear like analysis on nonlinear circuit is effective. That is why the fixator-norator pair (FNP) getting attraction.

A FNP can fix a critical biasing spec of an active device at a desired point on the characteristic curve. Thus the additivity property of superposition is applicable in such circuits where each critical operating point is fixed using appropriate FNPs. A fixator is a nullator plus source(s). FNPs are realized to use in analog design using ideal controlled sources with very high gain, but in this paper, we use operational amplifiers. The former one can be used only in circuit simulators but the later one can be used in both simulators and practical labs. Thus such a study gains pedagogical importance.

Verhoeven et al. [1] proposed a method in which transistors are DC isolated with the help of controlled sources. These transistors are biased only at the end using local biasing sources. A similar methodology is presented by Reza Hashemian [2] called 'local biasing'. Here no controlled sources are used for biasing purpose but the total number of local biasing source are reduced to half by replacing other half using storage elements like inductors and capacitors. The major difficulty with local biasing is the large number of scattered supplies. Source transformation methods can be used to reduce their number, but such processes are tedious and time consuming. Later, Reza [3]-[5] introduced 'fixator-norator pair', which can avoid many of the problems associated with the earlier methods. Fixators and norators are always used in pair and their use is temporary in the circuit. They can be replaced with actual circuit components when the design process is completed. Circuit simulators cannot directly model the pair; hence in his works, ideal controlled sources with very high gain are used to represent fixator-norator pairs.

The remaining part of this paper is arranged as follows. Section II introduces op-amp fixator and its working. A comparison is also made between op-amp fixator and its theoretical counterpart. Section III gives the case study with three examples. Finally section IV concludes the methodology.

\section{OPERATiOnAl AMPlifiers as Fixators}

Op-amp is a perfect nullor. That means, its input port acts as a nullator and its output port act as a norator. The output of op-amp can take any value so that its input is zero. This distinctive feature allows us to construct opamp voltage fixator and current fixator as shown in Fig. 1.

Fixator realized using controlled sources produce the values of power conducting components based on a given bias source. The op-amp fixator [6] gives solutions that are useful to generate the values of bias supporting components independent of the value of bias source. In Fig. 1 (a) and (b), fixators fix voltage between nodes X and $\mathrm{Y}$ at $\mathrm{V}_{\mathrm{F}}$ volt. In Fig. 1(c) and (d), fixators fix current from $\mathrm{X}$ to $\mathrm{Y}$ to be $\mathrm{I}_{\mathrm{F}}$ ampere. To understand the working of op-amp fixators, consider the circuits shown in Fig. 2. 


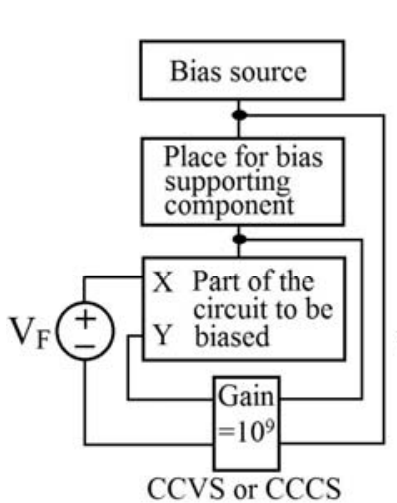

(a)

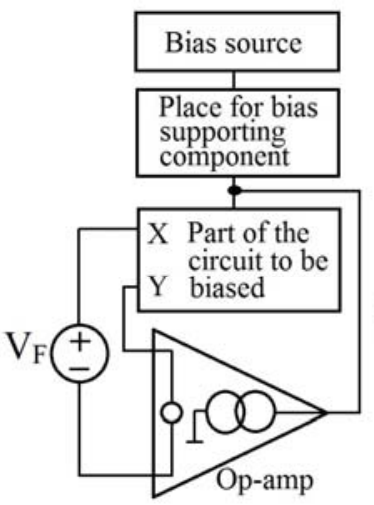

(b)

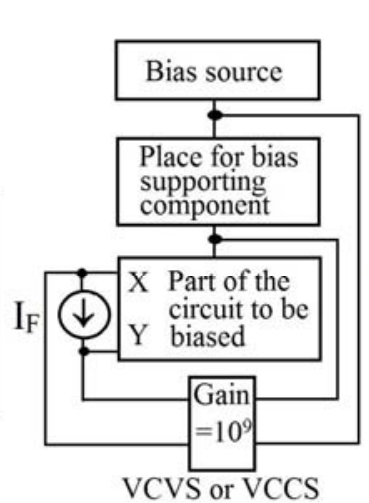

(c)

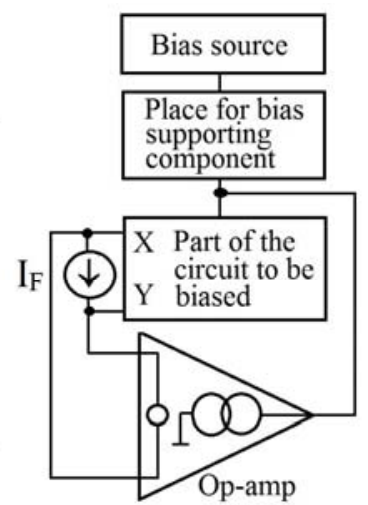

(d)

Fig. 1. Usage of voltage fixator; (a) using controlled sources; (b) using op-amp; and current fixator (c) using controlled sources; (d) using op-amp.

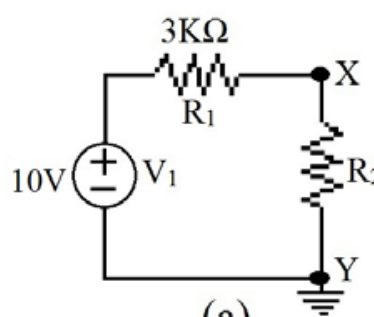

(a)

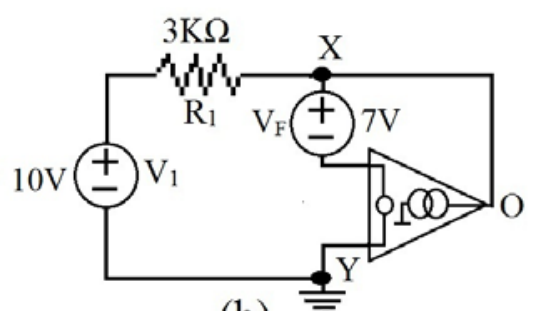

(b)

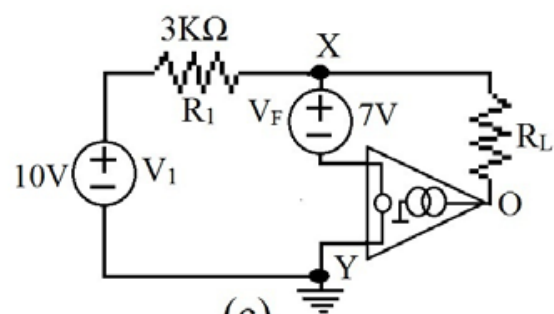

(c)

Fig. 2. (a) A simple resistor circuit; (b) applying voltage fixator of 7V; (c) circuit is modified as a current source.

Consider Fig. 2 (a), our aim in this experiment is to find the value of $R_{2}$ so that the voltage at node $\mathrm{X}$ will be $7 \mathrm{~V}$. We can apply op-amp fixator into the circuit as shown in Fig. 2(b). Here, $R_{2}$ is removed and instead op-amp fixator is added. The output port of op-amp does the role of bias supporting component to get $7 \mathrm{~V}$ across node $\mathrm{X}$. Simulation shows that current sink by the norator is $1 \mathrm{~mA}$, therefore $\mathrm{R}_{\mathrm{F}}$ should be $7 \mathrm{~V} / 1 \mathrm{~mA}=7 \mathrm{~K} \Omega$. Op-amp adjusts its output to maintain 'zero input' at its input port. Consider Fig. 2(c), a load resistor is inserted between node $\mathrm{X}$ and output of op-amp. It is a resistor in series with the current source (sink) and has no effect even if the load is increased, until the drop across the load reaches $V_{\mathrm{F}}$ plus saturation voltage of op-amp. Therefore, up to this limit, the circuit behaves like a current source and constant current of $1 \mathrm{~mA}$ flows through the load. These properties make the op-amp an ideal candidate to realize the fixators. A fixator $\operatorname{Fx}(0,0)$ is a nullor. Consider the inverting and non-inverting amplifier circuits as shown in Fig.3. Here nullor model of op-amps are used.

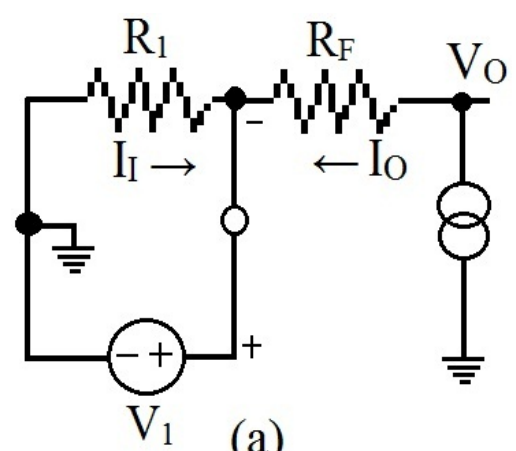

(a)

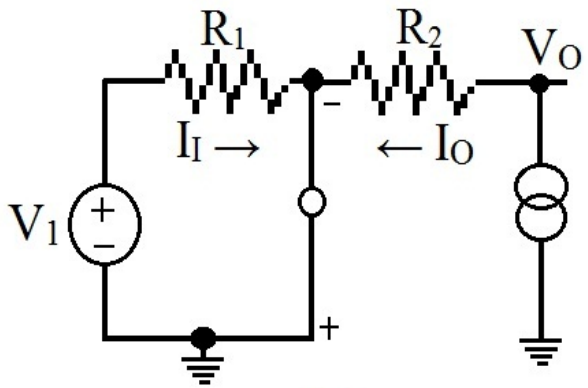

(b)

Fig. 3. (a) Non-inverting amplifier; (b) inverting amplifier.

We can derive the output equations for these amplifiers in a more easy way. This is the advantage of using fixators in analog circuits. 


\section{CASE STUdy}

Op-amp fixator find applications in source allocation, biasing design, fault detection etc., some of them are demonstrated in following example problems. Fig. 4 shows the process of fault identification and correction process of analog circuits using fixator.

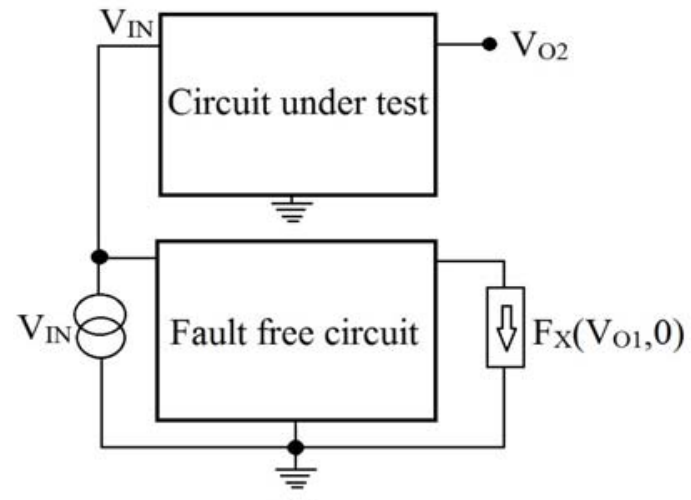

(a)

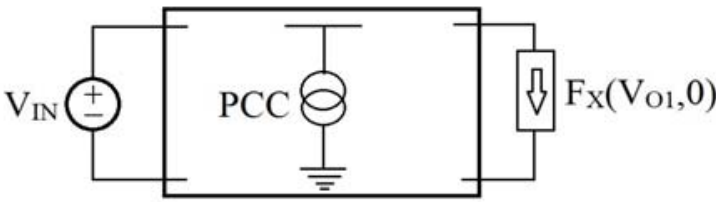

Faulty circuit

(b)

Fig.4. (a) Fault identification; (b) correction.

With a fault free circuit, in Fig. 4(a), a fixator $\mathrm{Fx}\left(\mathrm{V}_{\mathrm{O} 1}, 0\right)$ a known output, applied at the output of a fault free circuit produce the required input across the norator connected at the input port, for generating an output $\mathrm{V}_{\mathrm{O} 1}$. With the arrangement as shown in Fig. 4(a), the outputs $\mathrm{V}_{\mathrm{O} 1}=\mathrm{V}_{\mathrm{O} 2}$ if the circuit under test is fault free, otherwise the output $\mathrm{V}_{\mathrm{O} 2}$ deviates from $\mathrm{V}_{\mathrm{O} 1}$. For a faulty circuit, fixator can be applied to redesign the value of some of the power conducting components (PCCs) to eliminate the fault, as shown in Fig. 4(b).

Problem 1: Consider the scaling amplifier circuit [7] as shown in Fig. 5(a). Originally it was designed to give an output voltage of the form $v_{0}=8 v_{a}+6 v_{b}+3 v_{c}-7 v_{1}-v_{2}-5 v_{3}$. But due to the variation of one of the PCCs, say $R_{y}$, the output shifted from its designed value. In this case, op-amp fixator can be employed to re-design the appropriate value for $\mathrm{R}_{\mathrm{y}}$ and the designing process is shown in Fig. 5(b).

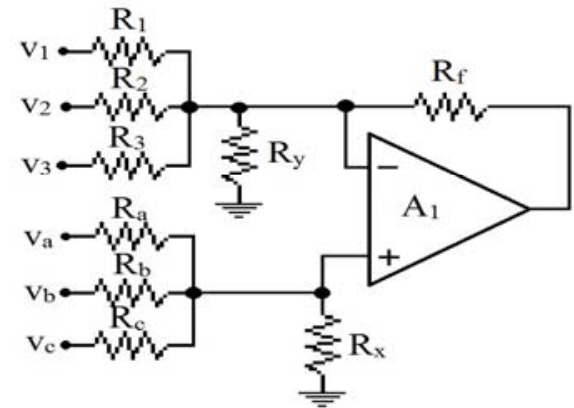

(a)

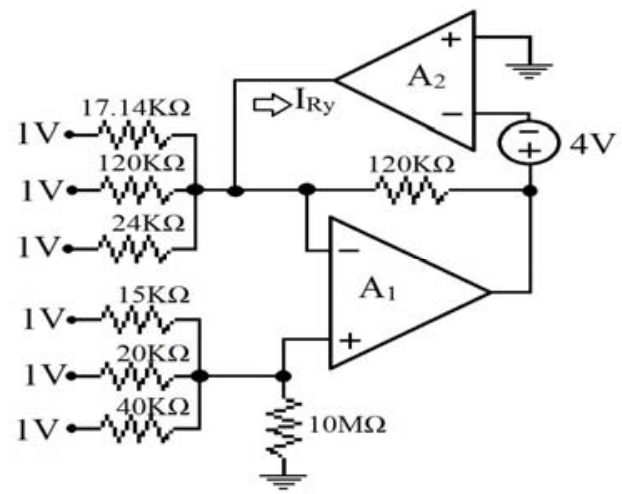

(b)

Fig.5. (a) Scaling amplifier; (b) re-designing $\mathrm{R}_{\mathrm{y}}$ for a given output expression.

Taking inputs $\mathrm{v}_{1}, \mathrm{v}_{2}, \mathrm{v}_{3}$ and $\mathrm{v}_{\mathrm{a}}, \mathrm{v}_{\mathrm{b}}, \mathrm{v}_{\mathrm{c}}$ as $1 \mathrm{~V}$, to satisfy the given output expression, we need an output of 4 volt. Therefore in Fig. 5(b), op-amp fixator $\mathrm{V}_{\mathrm{O}}(4 \mathrm{~V}, 0)$ is applied to keep the output voltage at $4 \mathrm{~V}$ and at the same time, output port of op-amp, the norator plays the role of $R_{y}$. Simulating the circuit indicates that $V_{R y}=1 V$ and $I_{R y}=25 \mu \mathrm{A}$. Therefore new value for $R_{y}=40 \mathrm{~K} \Omega$. It is now necessary to verify the result, so replace op-amp fixator in Fig. 5(b) with a $40 \mathrm{~K} \Omega$ resistor, and simulate the circuit. The output is $4 \mathrm{~V}$ and the result is verified.

Problem 2: Our aim in this problem is to design a basic BJT current source circuit having dynamic impedance $\mathrm{r}_{\mathrm{m}}=55 \mathrm{~K} \Omega$ and static impedance $\mathrm{R}_{\mathrm{M}}=2 \mathrm{~K} \Omega$. The circuit consists of two transistors and a resistor as shown in Fig. 6(a). This circuit is to be used as the active load for a common emitter amplifier as shown in Fig. 6(b). In this problem, op-amp fixator is used to design the current source as per the design requirement. 


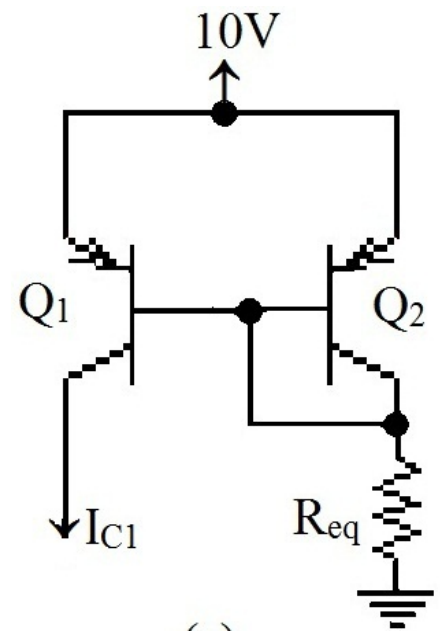

(a)

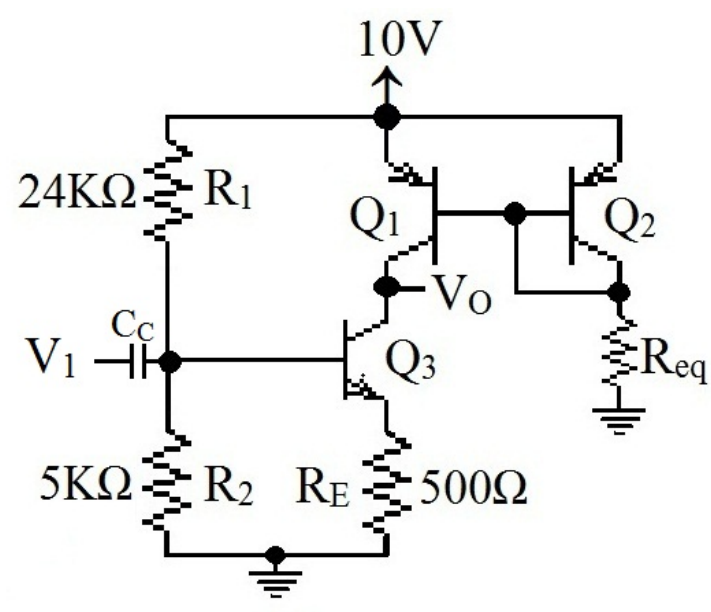

(b)

Fig. 6. (a) Current source; (b) common emitter amplifier with current source load.

The essential parameters for the active load, $V_{M}, I_{M}, R_{M}$ and $r_{m}$ are $4 V, 2 m A, 2 K \Omega$ and $55 \mathrm{~K} \Omega$ respectively, so that the voltage gain of the amplifier will be greater than 100 .

Op-amp fixator can be used to design the current source to meet the parameters as shown above. The design process is shown in Fig. 7(a). Early voltage VA for the identical transistors $\mathrm{Q}_{1}$ and $\mathrm{Q}_{2}$ is $110 \mathrm{~V}$, so that the dynamic resistance of the active load is $\mathrm{VA} / \mathrm{I}_{\mathrm{m}}=55 \mathrm{~K} \Omega$. Simulation shows that the value of $\mathrm{R}_{\mathrm{eq}}$ is $4.7 \mathrm{~K} \Omega$. The output waveform of the updated circuit for an input $\operatorname{Sin}(2000 \pi \mathrm{t}) \mathrm{mV}$ is shown in Fig. 7(b).

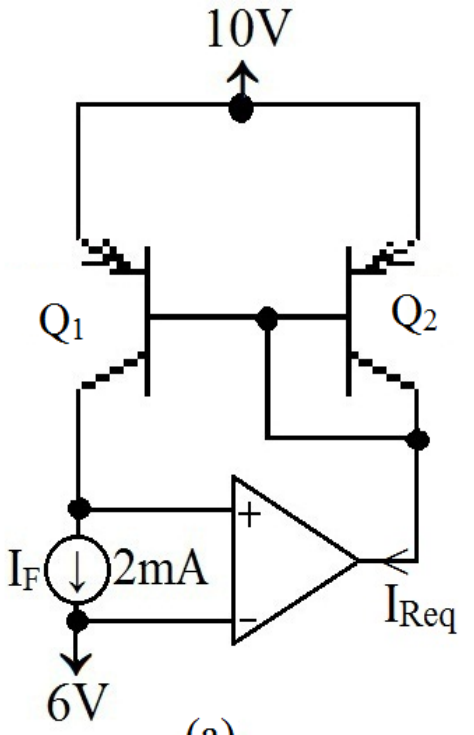

(a)

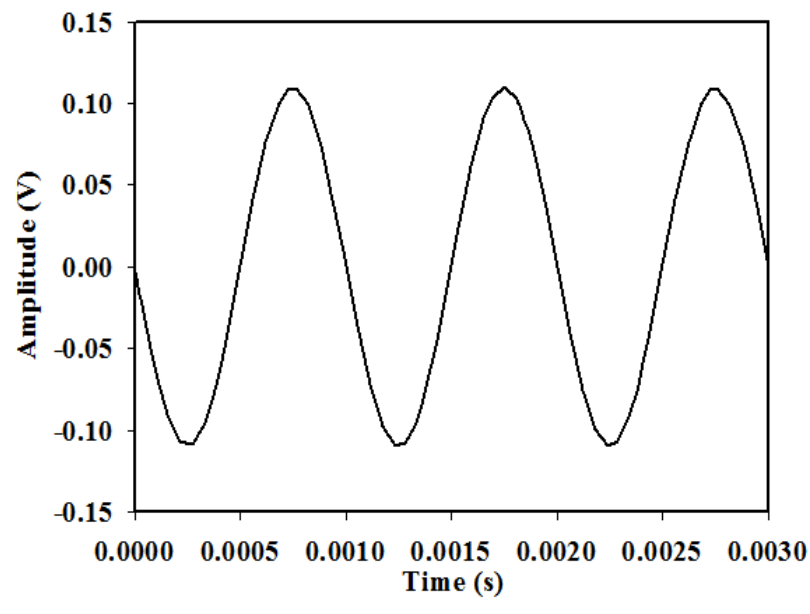

(b)

Fig. 7(a) design of current source; (b) output of the updated common emitter amplifier.

The small signal analysis of the common emitter amplifier is shown below. The output resistance is close to our design of $55 \mathrm{~K} \Omega$.

\section{$* * * *$ SMALL-SIGNAL CHARACTERISTICS}

INPUT RESISTANCE AT V_V1 $=4.138 \mathrm{E}+03$

OUTPUT RESISTANCE AT V(VO) $=5.339 \mathrm{E}+04$

Problem 3: Fig. 8(a) shows a well designed CMOS differential amplifier [8] with buffer stage. However, replacement of some of the components with similar devices disturbed the AC performance design of the amplifier. The objective here is to fix the AC gain of the amplifier at a desired value by re-designing the current source $\mathrm{I}_{\mathrm{S}}$ for its dynamic impedance. The design process begins with creating the linear model of CMOS amplifier as depicted in Fig. 8(b). Here, $r_{1}, r_{2}$ and $r_{3}$ represent the dynamic impedance of active loads $R_{1}, R_{2}$ and $\mathrm{R}_{3}$ respectively, $r_{\mathrm{gs}}$ represents extremely high resistance or open circuit. For a fixed input of $10 \mathrm{mV}$, the amplifier must produce an output of $3.17 \mathrm{~V}$ so that the gain of the amplifier is $50 \mathrm{~dB}$. Therefore, an op-amp fixator vo($3.17 \mathrm{~V}, 0)$ is added at the output port of the amplifier. To match the fixator applied, there should be a norator in 
the circuit. This suggests to replace $\mathrm{r}_{\mathrm{is}}$, the resistor corresponding to dynamic resistance of $\mathrm{I}_{\mathrm{S}}$ with the output terminal of op-amp as shown in Fig. 8(b). Now, we are able to simulate this circuit, the simulation shows that $r_{i s}=56 \mathrm{~K} \Omega$. Finally, the op-amp fixator is removed and the value of $r_{i s}$ is updated to $56 \mathrm{~K} \Omega$. The small signal analysis of the redesigned circuit is shown below, which is almost close to the expected gain.
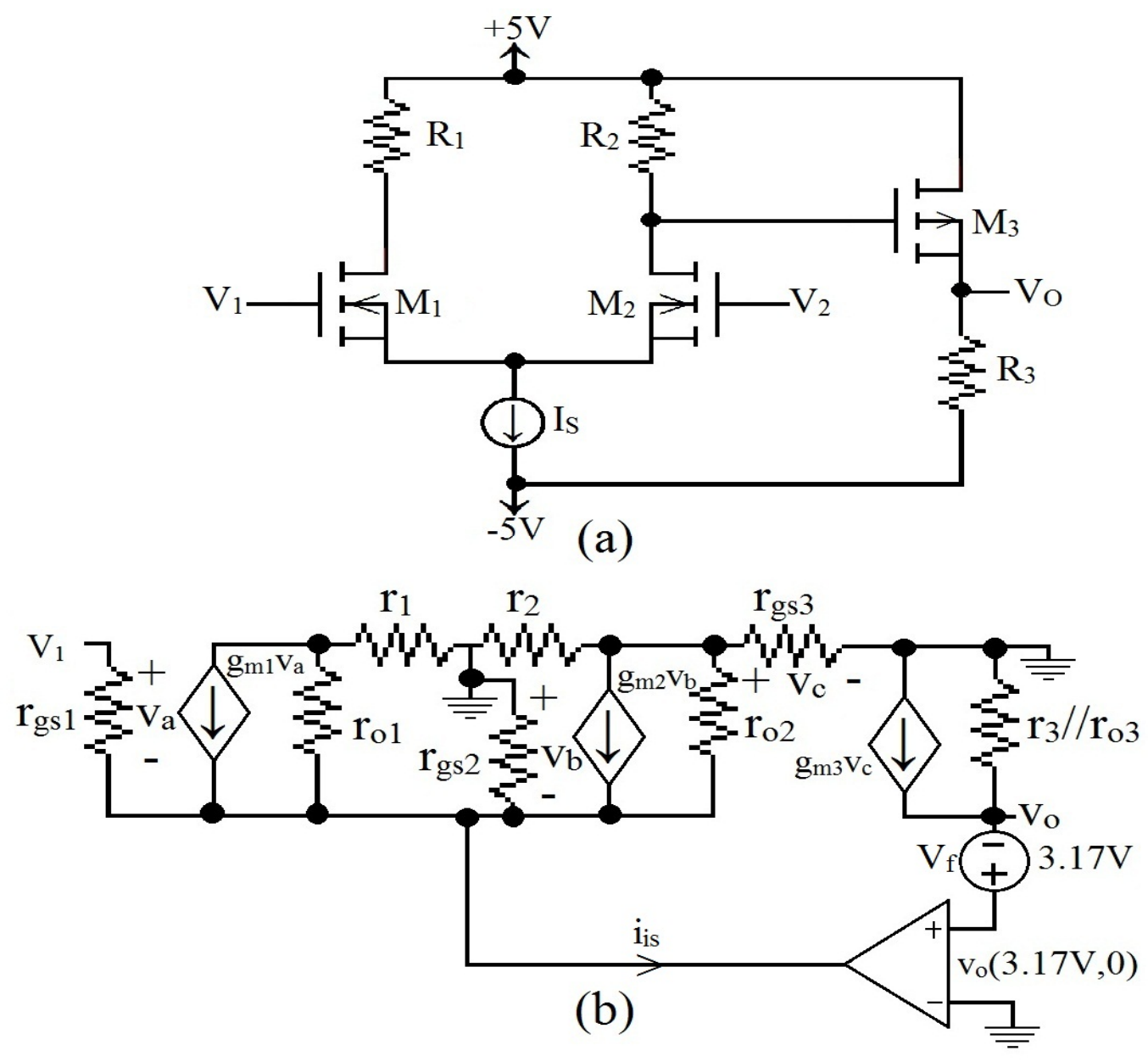

Fig. 8. (a) CMOS amplifier with buffer stage; (b) Design of $\mathrm{r}_{\mathrm{is}}$ using op-amp fixator.

\section{$* * * * \quad$ SMALL-SIGNAL CHARACTERISTICS}

$\mathrm{V}(\mathrm{VO}) / \mathrm{V}_{-} \mathrm{V} 1=-3.163 \mathrm{E}+02$

INPUT RESISTANCE AT V_V1 $=1.000 \mathrm{E}+20$

OUTPUT RESISTANCE AT V(VO) $=2.449 \mathrm{E}+04$

\section{CONCLUSION}

A method for the realization of fixator-norator pair has been presented. The op-amp fixators are proved to be a good alternative for controlled sources. Three applications of the op-amp fixators, a scaling amplifier, an active load for common emitter amplifier and design of a CMOS differential amplifier are explained in detail and the results are verified by simulation. The results are in close proximity with the design.

\section{ACKNOWLEDGMENT}

Authors are thankful to UGC, Government of India for providing necessary fund to do this research work. 


\section{REFERENCES}

[1] C. J. Verhoeven, A. van Staveren, G. L. E. Monna, M. H. L. Kouwenhoven, and E. Yildiz, Structured Electronic Design: NegativeFeedback Amplifiers, Kluwer Academic Publishers, Dordrecht, The Netherlands, 2003

[2] R. Hashemian, "Local biasing and the use of nullator-norator pairs in analog circuits designs", VLSI Design vol. 2010, pp.1930, 2010.10.1155/2010/297083 [Online]. Available: http://www.hindawi.com/journals/vlsi/2010/297083.html

[3] Reza Hashemian, "Application of Fixators-Norator Pairs in Designing Active Loads and Current Mirrors in Analog Integrated Circuits", IEEE Transactions On Very Large Scale Integration (VLSI) Systems, Vol. 20, No. 12, December 2012.

[4] E. Tlelo-Cuautle, Advances in Analog Circuits, 1st ed. India: InTech, 2011.

[5] Reza Hashemian, "Fixator-Norator Pair versus Direct Analytical Tools in Performing Analog Circuit Designs", IEEE Transactions on Circuits and Systems-II: Express Briefs, Vol.61, No. 8, August 2014.

[6] Rohith Krishnan R, S Krishnakumar, Reza Hashemian, "A Method to Modify/Correct the Performance of Amplifiers," IJSTR, ISSN: 2277-8616,Vol.4,Issue 1, January 2015.

[7] Muhammad H. Rashid, Microelectronic Circuits Analysis and Design, Second Edition, Cengage Learning Inc, 2011.

[8] R. Hashemian, "New Port Modeling for Analog Circuit Biasing Design", 2010 IEEE International Conference on Electro/Information Technology EIT2010, Normal, IL, May 13 - 15, 2010.

\section{AUTHOR PROFILE}

R. Rohith Krishan completed his B.Sc. in Electronics from Mahatma Gandhi University, Kottayam in 2011 and M.Sc. in Electronics with first rank from Mahatma Gandhi University, Kottayam in 2013. He was awarded the prestigious NET-JRF by UGC, Government of India in October, 2013. Presently he is working as a Junior Research Fellow at the Department of Electronics, STAS, Mahatma Gandhi University Regional Centre, Edappally, Kochi-24, Kerala, India. His research interest fields are Analog circuit design, Fixator-norator pair and fixator based designs.

S. Krishnakumar completed his M.Sc. in Physics with Electronics specialization in 1987 from Mahatma Gandhi University, Kottayam. He was awarded Ph.D. in Thin Film Devices in 1995 from Mahatma Gandhi University, Kottayam. He got M.Tech. in Computer Science from Allahabad Agricultural Institute - Deemed University (renamed as Sam Higginbottom Institute of Agriculture, Technology and Sciences) in 2006 and also obtained MCA from IGNOU in 2010. He has 21 years of teaching experience in Electronics and Computer Science subjects for graduates and post graduates. Currently he is working as the Regional Director of School of Technology and Applied Sciences (STAS), Mahatma Gandhi University Regional Centre, Edappally, Kochi-24, Kerala. India. His research interest fields include Analog circuit design, VLSI and Image processing.

Dr. S. Krishnakumar is an Associate member of Institute of Engineers, India. He was a member of Board of studies of University of Calicut and a member of Academic council of Mahatma Gandhi University, Kottayam. 